\section{Has Physics Discarded Mechanism ?}

IN his British Association presidential address of 1931, General Smuts pointed out that "the older mechanistic conception of Nature is being modified". This is undeniable; but to what extent does it justify the accompanying statement that "the very basis of mechanism is undermined"'? This has been repeated frequently, with applications to psychology, ethics and philosophy that are as obvious as they are debatable.

What then is the meaning of 'wave mechanics' and 'quantum mechanics'? If we define mechanisms as systems, of any kind, in which their constituent parts or factors co-operate definitely, precisely, and in most cases repetitively, in such a way that energy is transmitted, transferred, or transformed, is not some such concept not merely legitimate, but indispensable to physics, as distinct from pure mathematics, which may, or even must, dispense therewith ?

To cite specific examples, are not the rotating galaxy, the solar system, crystal oscillators, vibrating atoms, and possibly also electrons and protons, mechanisms in the same sense as a clock is a mechanism ? Further, if we regard any series of periodic changes as a wave series, may we not include under 'wave' a swinging pendulum, a water wave, and an electron?

It should be observed that purpose, in any form, is neither implied nor excluded, while the uncertainty principle may express only the limitations of knowledge, not necessarily the character of Nature.

Is not the 'older mechanistic conception' being replaced by the concept of mechanisms which are so much more complex, delicate and precise than earlier models that only mathematical expressions are adequate for their description and explanation ? But it does not follow that, because such mechanisms cannot be imaginatively represented, they are therefore non-existent; it simply implies that they are too complicated for imagination to grasp. The advance in theory, then, does not justify the complete discarding of mechanism, as such.

The University of Liverpool,

J. E. TURner. Aug. 22.

\section{Change of Paramagnetic Susceptibility due to Absorption of Light}

WE have already published in NATURE ${ }^{1}$ a note giving the results of our observation on the change in susceptibility produced in paramagnetic solutions like that of chromic chloride, etc., due to light absorption. Following the suggestion of S. Kato, we had assumed that the absorption maxima at $\lambda 4300$, 6100 in a given solution of chromic chloride were due to the transition of the $\mathrm{Cr}+++$ ion from the ${ }^{4} F^{\prime}$ to ${ }^{2} G$ resp. ${ }^{2} H$ state. On the supposition that both in the initial and in the final state the magnetic moment of the ion is due only to the spin moments of the magnetic electrons, such transitions would be accompanied by diminution in the magnetic moment of the ion. We reported that the result of our observation indicated a diminution in the susceptibility of the chromic chloride solution and therefore was in agreement with our theoretical assumptions.

Recently Gorter ${ }^{2}$ reports that he has observed a similar diminution of susceptibility in a solution of chromic chloride due to absorption of light, but he finds that this diminution is to be attributed to rise in the temperature of the solution. Since the publication of our letter in NATURE, we have found that the deflection of the bulb containing the chromic chloride solution corresponded to an increase in its paramagnetic susceptibility and not to a diminution. A paper containing the results of our investigation was sent about two months ago to the Zeitschrift für Physik, where the experimental details will be found. We may mention here that we used a torsion balance of period 3-5 sec., and neutralised the effect of the field on the bulb containing the concentrated solution, by attaching a bismuth ball of suitable size below it. The response to the incident light was almost instantaneous, and after a prolonged exposure the light spot gradually returned to its original position and then moved over to the opposite side. Specchia $^{3}$ has also observed this quick inerease in magnetic susceptibility due to light absorption.

In using the torsion balance, the bulb has to be placed almost in a position of instability in the inhomogeneous magnetic field, and for each set of observations a new adjustment has to be made. Under such conditions it is difficult to obtain reproducible results. We have, therefore, tried other methods of making this effect measurable. The following one we think will give fairly consistent results. A modified form of the inclined capillary tube arrangement used by Liebknecht and Wills" is filled with chromic chloride solution and the meniscus is placed between the pointed poles of an electromagnet. Light from a mercury arc, after passing through an infra-red filter, is focused from the top on the meniscus. Observation of the position of the latter in the magnetic field is made, once when the incident light from the arc is further filtered through a concentrated solution of chromic chloride, and again when this filter is taken off. Deflections of about 1.5 scale divisions of the micrometer scale in the eye-piece of the observing telemicroscope, indicating an increase of susceptibility, were noted.

Since the transitions from the $4 F^{\prime}$ to the ${ }^{2} G$ resp. the ${ }^{3} \mathrm{H}$ state represent diminution of the spin moment of the ion, the observed increase in susceptibility can only be interpreted as being due to the temporary break down of the $l$-coupling between the $\mathrm{Cr}+++$ ion and the associated water molecules. Some interesting applications of this result are given in another paper which appears in the Zeitschrift für Physik.

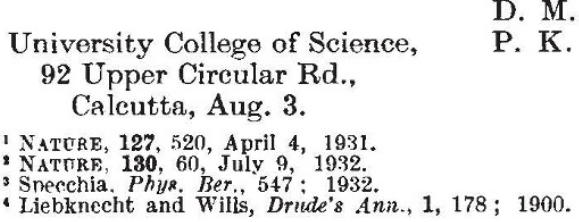

\section{Iodine in Cod Liver Oil}

The purpose of this note is to indicate to British workers in the field of endocrine secretions and nutrition certain experimental work which we have thus far withheld from complete publication pending studies which we are now ready to report in detail.

We showed ${ }^{1}$ that ferrous iodide mixed with the food would benefit rats on a diet deficient in vitamin A. Later we demonstrated that unsaturated fatty acids were apparently indicated in addition. ${ }^{2}$ More recently we have summarised our findings. ${ }^{3}$

Studies made during the past winter and spring in which we induced leg-weakness in chicks by the intravenous injection of anterior pituitary extract, but prevented it in other animals by orally adminis.

No. 3284, VoL. 130] 